Original

\title{
Prevalencia de infeccion latente tuberculosa en trabajadores sanitarios
}

\section{Prevalence of the latent tuberculosis infection in sanity workers}

Brígido Pérez Bermúdez , Manuel Antonio Aranda Vizcaíno, Pablo Honorio Labanda Urbano.

Hospital de La Princesa. Servicio de Prevención de Riesgos Laborales. Área 2. Madrid. España.

Recibido: 01-02-11

Aceptado: 22-02-11

\section{Correspondencia}

Brígido Pérez Bermúdez

Hospital de La Princesa.

Servicio de Prevención de Riesgos Laborales. Área 2

Diego de León 62

28006 Madrid

Tfno: 915202596

bperezb.hlpr@salud.madrid.org

ResUmen

Introducción: En el ámbito laboral sanitario la Tuberculosis (TBC) es una enfermedad considerada de elevado riesgo para la salud de los trabajadores.

Objetivos: El objetivo fundamental de este trabajo consiste en estudiar la prevalencia de Infección Latente Tuberculosa (I.L.T.) en dos centros hospitalarios del Área 2 de Madrid y la distribución de esta I.L.T. entre las categorías y los servicios clínicos.

Material y métodos: Estudio transversal. La población analizada ha sido de 455 trabajadores sanitarios de los Hospitales Universitarios de La Princesa e Infantil del Niño Jesús. El marco temporal del estudio abarca desde los años 2006 a 2009. Las variables analizadas han sido la edad, el sexo, tipo de hospital, la categoría profesional y los servicios clínicos.

Resultados: La prevalencia de I.L.T observada ha sido del $11.4 \%$ ( $8.3 \%-14.1 \%$ ). No se han encontrado diferencias significativas de I.L.T. entre los distintos centros sanitarios estudiados, servicios clínicos, categorías y sexo. Sí es significativa la edad con una Odds Ratio de 1.05 (1.02-1.08).

Conclusiones: En nuestra área sanitaria la I.L.T en los trabajadores sanitarios es de un 10\% aproximadamente, y esta no tiene relación con los puestos de trabajo sino con la edad.

Med Segur Trab (Internet) 2011; 57 (222) 34-40

Palabras Claves: Infección Latente Tuberculosa, Mantoux en trabajadores sanitarios. Tuberculosis.

Abstract

Introduction: In the health sector, Tuberculosis is considered as a high risk disease for workers.

Objectives: The main objective of this report is to study the prevalence of the Latent Tuberculosis Infection (I.L.T.) in two hospitals of the Area 2 of Madrid and the distribution of this infection among the clinical categories and services. 
Material and methods: Transversal study. We have analyzed 455 health workers of the University Hospital "La Princesa" and children's Hospital "El Niño Jesús". The study began in 2006 and ended in 2009. The analyzed variables have been age, sex, type of hospital, professional category and clinical services.

Results: The prevalence of the observed latent tuberculosis infection has been of $11.4 \%(8.3 \%-14.1 \%)$. No significant differences have been observed between type of hospital, clinical services, professional category and sex. Age has been pointed out as a significant factor with an Odds Ratio of 1.05 (1.02 - 1.08).

Conclusions: In our area of study the I.L.T. in health workers is approximately 10\%, and this is not related with professional category but with age.

Med Segur Trab (Internet) 2011; 57 (222) 34-40

Key Words: Latent Tuberculosis Infection, Skin testing for tuberculosis in bealtbcare workers, Tuberculosis. 


\section{INTRODUCCIÓN}

En el ámbito laboral sanitario la Tuberculosis (TBC) es una enfermedad considerada de elevado riesgo para la salud de los trabajadores, esto es debido fundamentalmente a que es una enfermedad de transmisión aérea y por las consecuencias que acarrearía no solo para el trabajador sino por las que produciría un sanitario enfermo y bacilífero para los pacientes que este atiende.

Encuadrado dentro de los Riesgos Biológicos es, junto con los virus de transmisión parenteral (Virus de la Hepatitis B, Hepatitis C y Virus de la Inmunodeficiencia Humana), el microorganismo que más atención y recursos mueve para su prevención.

La incidencia de enfermedad tuberculosa en España en el año 2008, fue de 14.54 casos / $10^{5}$ habitantes-año ${ }^{1}$. En el año 2009, para la Comunidad de Madrid la incidencia fue de 14.94 casos $/ 10^{5}$ habitantes-año ${ }^{2}$ registrándose en el Área 2 , a la que pertenecen los centros hospitalarios de este estudio, incidencias de 6.15 casos $/ 10^{5}$ habitantes-año. Estas cifras reflejan la baja incidencia de enfermedad en la población atendida por los profesionales sanitarios de nuestros hospitales.

Existe mucha información sobre indicadores de enfermedad tuberculosa en la población, ya que al ser considerada enfermedad de declaración obligatoria por sus repercusiones en la salud pública, los registros son extensos y fiables. No ocurre lo mismo con los datos referentes a la Infección Latente Tuberculosa (I.L.T.). En los últimos años existen algunos trabajos en los que se estudia la prevalencia de I.L.T. en poblaciones de inmigrantes al proceder de países con elevadas tasas de incidencia de enfermedad tuberculosa, haciendo aconsejable su estudio para el despitaje de enfermedad. La I.L.T en algunos colectivos inmigrantes como los provenientes de África y Latinoamérica encuentran prevalencias de hasta un $40 \%{ }^{3,4,5}$.

En el mundo laboral sabemos que existen ciertos colectivos que están más expuestos al bacilo tuberculoso, como son el personal de centros de acogida de población inmigrante, cuidadores de población reclusa, personal que trabaja con adictos a drogas por vía parenteral, trabajadores sanitarios, etc, etc.

Concretamente para los trabajadores sanitarios se considera enfermedad profesional y existen diferentes trabajos no solo en España ${ }^{5}$, sino en otros países que analizan y constatan un mayor riesgo de enfermedad entre los trabajadores sanitarios, aunque parece que es más probable en países con elevada incidencia ${ }^{6,7,8 .}$

El objetivo fundamental de este trabajo consiste en estudiar la prevalencia de I.L.T. en dos centros hospitalarios del Área 2 de Madrid con el fin de conocer nuestros valores de referencia así como la prevalencia para comparar con otros hospitales y Áreas sanitarias. También se analiza si la prevalencia es más alta entre los trabajadores sanitarios más expuestos según edad, sexo, categoría laboral, servicios clínicos y centro de trabajo, como en algunas bibliografía parece indicar.

\section{MATERIAL Y MÉTODOS}

Se ha realizado un estudio transversal. La población analizada han sido 455 trabajadores sanitarios de los Hospitales Universitarios de La Princesa e Infantil del Niño Jesús. El marco temporal del estudio abarca desde los años 2006 a 2009.

Hemos considerado I.L.T. cuando la prueba del Mantoux era positiva. Esta se realizó a los trabajadores dentro de los procedimientos de Vigilancia de la Salud. Se utilizó el derivado proteico (PPD) RT-23, a dosis de 2 UT por $0.1 \mathrm{ml}$, considerando positivo todo Mantoux cuya induración fuese superior a $5 \mathrm{~mm}$ en el caso de que el trabajador no estuviese vacunado de BCG. Si el trabajador estaba vacunado, se consideró positivo cuando la induración fue superior a $14 \mathrm{~mm}$. En el caso de que el sanitario tuviese más de 55 años se realizó el efecto de refuerzo (Booster) a los 7 días, si el primer test fue negativo. A los 
trabajadores con Mantoux positivos se les solicitó posteriormente radiografía de tórax para descartar enfermedad tuberculosa y se ofreció profilaxis medicamentosa.

Las variables a estudio han sido la edad, el sexo, tipo de hospital, la categoría profesional y los servicios clínicos. Hemos creado una variable en la que se agrupan servicios clínicos con alto riesgo de contagio por atender con más probabilidad enfermos bacilíferos o se utilizan técnicas donde es más probable la transmisión del bacilo, denominándolos "servicios de alto riesgo". Se incluyen, Neumología, unidades de enfermedades infecciosas de Medicina Interna, Otorrinolaringología, Urgencias, Microbiología, Aerosolterapia, Broncoscopía y Anatomía Patológica.

Para el análisis estadístico se aplicó en la comparación de proporciones el ji cuadrado y para variables continuas la t-student. Posteriormente se realizó un análisis multivariante (Regresión Logística) con el fin de detectar variables confusoras y construir un modelo predictivo. La medida de asociación fue una Odds Ratio (O.R.) y se calcularon sus Intervalos de Confianza al $95 \%$ (I.C. 95\%).

\section{RESULTADOS}

De un total de 455 Mantoux realizados entre los trabajadores sanitarios del Hospital U. de La Princesa y del H. U. Infantil Niño Jesús, encontramos una prevalencia de I.L.T de $11.4 \%(8.3 \%-14.1 \%)$

Los resultados de la prevalencia de infección de las distintas variables así como el resultado del análisis bivariante se refleja en la tabla I.

TABLA I. Resultados de Mantoux Positivos según variables analizadas.

\begin{tabular}{|c|c|c|c|}
\hline VARIABLES & PPD POSITIVOS & PPD NEGATIVOS & $\mathbf{P}$ \\
\hline \multicolumn{4}{|l|}{ SEXO } \\
\hline Hombre & $8(9.5 \%)$ & $76(90.5 \%)$ & \\
\hline Mujer & $44(11.9 \%)$ & $327(88.1 \%)$ & 0.704 \\
\hline EDAD* & 43.8 años (13.7) & 36.2 años (13.2) & 0.000 \\
\hline \multicolumn{4}{|l|}{ CENTRO DE TRABAJO } \\
\hline H. U. de La Princesa & $35(9.9 \%)$ & $319(90.1 \%)$ & \\
\hline H. I. U. Niño Jesús & $17(16.8 \%)$ & $84(83.2 \%)$ & 0.074 \\
\hline \multicolumn{4}{|l|}{ SERVICIOS SANITARIOS } \\
\hline Servicios alto riesgo ${ }^{* *}$ & $3(4.5 \%)$ & $64(95.5 \%)$ & \\
\hline Médicas & $24(11.3 \%)$ & $188(88.7 \%)$ & \\
\hline Quirúrgicas & $9(11.1 \%)$ & $72(88.9 \%)$ & \\
\hline S. Centrales & $12(16.0 \%)$ & $63(84.0 \%)$ & \\
\hline Gestión-Cocina-Mantenimiento & $2(13.3 \%)$ & $13(86.7 \%)$ & 0.340 \\
\hline \multicolumn{4}{|l|}{ CATEGORIAS PROFESIONALES } \\
\hline Médicos & $14(8.0 \%)$ & $161(92.0 \%)$ & \\
\hline Enfermería / Fisioterapeutas & $19(15.1 \%)$ & $107(84.9 \%)$ & \\
\hline Aux. Enfermería /Técnicos & $12(10.2 \%)$ & $106(89.8 \%)$ & \\
\hline Celadores & $4(20.0 \%)$ & $16(80.0 \%)$ & \\
\hline Otros & $3(18.8 \%)$ & $13(81.3 \%)$ & 0.186 \\
\hline
\end{tabular}

Como puede observarse no existen diferencias significativas entre sexo, centro de trabajo, servicios clínicos y categoría profesional. Es importante destacar que existe una diferencia que es casi significativa $(\mathrm{p}=0.07)$ entre los trabajadores con Mantoux positivo de un centro y de otro. Así en el Hospital Infantil U. Niño Jesús existe una prevalencia de $16.8 \%$ y en el H. U. de La Princesa de $9.9 \%$. 
Posteriormente se realizó una Regresión Logística (R.L.) cuyos resultados se muestran en la tabla II.

TABLA II. Resultados de la Regresión Logística.

\begin{tabular}{lccc}
\hline \multicolumn{1}{c}{ VARIABLES } & P & OR & I. C. (95 \%) \\
\hline SERVICIOS SANITARIOS & & & \\
Alto riesgo** & & 1 & $0.60-7.62$ \\
Médicas & 0.23 & 2.15 & $0.48-7.70$ \\
Quirúrgicas & 0.35 & 1.92 & $0.53-9.43$ \\
S. Centrales & 0.26 & 2.26 & $0.10-10.8$ \\
Gestión-Cocina-Mantenimiento & 0.35 & 0.32 & \\
CATEGORIAS PROFESIONALES & & & \\
Médicos & & 1 & $0.49-3.12$ \\
Enfermería / Fisioterapeutas & 0.23 & 1.24 & $0.19-1.70$ \\
Aux. Enfermería /Técnicos & 0.35 & 0.57 & $0.05-5.51$ \\
Celadores & 0.26 & 0.55 & $0.23-101.55$ \\
Otros & 0.53 & 4.92 & $0.31-1.39$ \\
CENTRO DE TRABAJO & 0.27 & 0.66 & $0.49-2.88$ \\
SEXO & 0.70 & 1.19 & $\mathbf{1 . 0 2}-\mathbf{1 . 0 8}$ \\
EDAD & $\mathbf{0 . 0 0}$ & $\mathbf{1 . 0 5}$ & \\
\hline *** Servicios de alto riesgo =Urgencias, Infecciosas, Neumología,Aerosolterapia, Broncoscopía, Microbiología, Otorrinolaringología, \\
$\quad$ Necropsias
\end{tabular}

La probabilidad pronosticada al aplicar una curva ROC (Receiver Operating Characteristic) es de 0.74 (0.62 - 0.78), es decir con este modelo al elegir dos casos, uno con I.L.T. y otro sin I.L.T. se clasificarían correctamente en un $74 \%$ de las veces.

Como vemos en la tabla II, solo la edad es significativa. Por cada año de edad que tenga más el trabajador, aumenta un $5 \%$ la probabilidad (O.R) de que padezca una I.L.T.

El resto de las variables estudiadas no son significativas. La significación del centro de trabajo, que en el análisis bivariante se acercaba a la significación, al construir el modelo de regresión logística de esta variable, la significación no se confirma. Esto es debido a que la edad media de los trabajadores en el H. Infantil U. del Niño Jesús es mayor.

\section{DISCUSIÓN}

Estos resultados no deben ser generalizados. Posiblemente en otros hospitales que atiendan otras zonas y áreas geográficas las conclusiones puedan resultar distintas. Los enfermos atendidos con tuberculosis activa y bacilífera en los centros sanitarios depende de la prevalencia e incidencia de la Tuberculosis del área de atención de estos centros y en nuestro área es de las más bajas de la Comunidad de Madrid, como así lo indicamos en la introducción ${ }^{2}$.

Llama la atención la escasa literatura que existe actualmente sobre la prevalencia de I.L.T en los distintos colectivos laborales y en la población en general. En los últimos años se han realizado diferentes estudios en colectivos de inmigrantes y reclusos?.

La realización de estudios de prevalencia de I.L.T en los últimos años, ha sido propiciada por la implantación de la nueva técnica interferón-gamma (INF- $\bigotimes$ ), fundamentalmente estudios de sensibilidad-especificidad y de coste-beneficio, siendo la mayoría de las veces la población diana trabajadores sanitarios ${ }^{10,11,12}$. Los resultados obtenidos por esta técnica son mejores que con la técnica de intradermoreacción (Mantoux) ya que la especificidad es superior ${ }^{13}$. Entre otros argumentos se esgrimen que la técnica del INF- $\square$ detecta específicamente la I.L.T. discriminando a los trabajadores infectados por el M.Ttuberculosis de los que han recibido la vacunación y los que han sido expuestos a otras micobacterias ambientales. Otra ventaja añadida es que elimina la subjetividad de algunos 
resultados evitando la perdida de individuos que no acuden a las lecturas. Como desventaja parece que es una técnica de un mayor coste, con cierta complejidad en su realización y aún no implantada en todos los laboratorios. Aunque existen publicaciones que afirman que el coste final del estudio de la I.L.T. es similar utilizando ambas técnicas ${ }^{4,15,16}$.

En los resultados obtenidos se observa que no existen diferencias significativas entre los dos hospitales analizados, Hospital U. de La Princesa y el H. U. I. del Niño Jesús, a pesar de que en el estudio bivariante existe una $\mathrm{p}=0.07$. Al estudiar el modelo multivariante esta posible significación desaparece obteniendo una O.R de 1.14 (0.89 1.47). Esto es debido a que la edad de los trabajadores es mayor en el Hospital I. U. del Niño Jesús (la media es de 40.4 años con una desv. típica 12.8 años) que en el H. U. de La Princesa ( la media es de 36.1 años con una desv. típica 13.5 años). Al tener más edad los trabajadores del hospital infantil la prevalencia de I.L.T. es mayor. Podría deberse entre otras causas a que el hospital infantil es más solicitado en traslados (personal más antiguo), al ser considerado por los trabajadores que la atención a estos pacientes es más atractiva para su desarrollo profesional (pacientes que producen mayor respuesta emotiva, requieren más facilidad en su manejo, etc).

En cuanto a los servicios clínicos destacamos que considerando el basal como la agrupación de servicios de alto riesgo, no existen diferencias significativas con el resto de los servicios sanitarios y no sanitarios. En los servicios de Especialidades Médicas, Cirugías y S. Centrales se observa una O.R. mayor de I.L.T. que en los servicios de alto riesgo, aunque estas diferencias no son significativas. Una explicación podría ser que existe una pequeña probabilidad de infección ya que para que se produzca, se necesita un periodo prolongado de proximidad con el paciente bacilífero y por otra parte cuando se diagnostica o se sospecha enfermedad tuberculosa se instaura aislamiento aéreo (habitación cerrada, con recambio de aire de más de 6 renovaciones por hora y presión negativa) y se implantan de los procedimientos y equipos de protección para la atención a estos pacientes por parte de los trabajadores (EPIs correspondientes) ${ }^{17}$. También hay que considerar que, precisamente en estos servicios de mayor I.L.T. los trabajadores tienen más edad que en los servicios denominados de "alto riesgo", fundamentalmente Urgencias y Unidades de Infecciosas integrados por población trabajadora mas joven. Lo que llama la atención es que no existen diferencias significativas entre la I.L.T. en zonas y áreas con más atención a pacientes bacilíferos que en unidades clínicas en las que este tipo de pacientes es menos frecuente, como son las especialidades quirúrgicas y las que se han definido como "otros servicios médicos" (Digestivo, Nefrología, Hematología, etc.) lo que de alguna forma incide en que los procedimientos de aislamiento y las medidas de protección a los trabajadores son los correctos.

En el análisis de las categorías profesionales tampoco apreciamos significación. Tomando como basal la categoría médica, se observa que existe una O.R. superior de padecer I.L.T. en enfermeria y colectivos clasificados como "Otros" (administrativos, pinches, mantenimiento, etc) respecto a la categoría basal (Médicos) pero sin ser significativas. Esta variable que analiza las categorías también está influenciada como en las demás, por la edad. En la categoría agrupada como "Otros", además de ser trabajadores con más edad, la muestra es escasa como lo demuestra el intervalo de confianza tan amplio (0.23-101.55).

Ya hemos apuntado que la edad es la única variable significativa. Se puede argumentar que posiblemente hubiese sido más conveniente registrar la antigüedad profesional o en los puestos de trabajo, pero en los centros sanitarios existe con mucha frecuencia una gran movilidad interna en algunas categorías (enfermería, aux. de enfermería y celadores) rotando en ocasiones por diversos servicios en una misma jornada laboral. Por otra parte al construir el modelo multivariante, el utilizar conjuntamente antigüedad y edad daría problemas de colinealidad.

Por último quisiéramos puntualizar que en el estudio de estas variables -Categorías y Servicios- hemos constatado que no existen diferencias significativas, al contrario de lo que otros estudios concluyen ${ }^{18,19}$, que si observan diferencias significativas en las 
categorías profesionales. Posiblemente esto sea debido a que en áreas geográficas con mayor incidencia de tuberculosis y con condiciones higiénicas, ambientales y laborales (aislamientos, epis, etc) mas deficitarios sean mas deficitarias.

Por tanto debemos concluir que en nuestros centros sanitarios y con la incidencia disminuyendo en los últimos 10 años ${ }^{1}$, no existe más riesgo de I.L.T. entre las distintas categorías profesionales ni en los diferentes servicios clínicos estudiados.

\section{REFERENCIAS BIBLIOGRÁFICAS}

1. E. Rodríguez, G. Hernández, S. Villarrubia, O. Tello. Casos de tuberculosis declarados a la Red Nacional de Vigilancia Epidemiológica. España 2008. Bol. Epidemiol Semanal 2009;17: 121-132.

2. Boletín Epidemiológico de la Comunidad de Madrid. 2010; $\mathrm{n}^{\circ} 1 ; 16: 71-73$.

3. Xavier Vallès, Francesca Sánchez, Helena Pañella, Patricia García de Olala, Joseph Maria Jansà y Joan A. Calayà. Tuberculosis importada: una enfermedad emergente en países industrializados. Med Clin (Bar) 2002; 118(10):376-8

4. J.M. Ramos a, C. Pastor b, M. Masia a, E. Cascales c, G. Royo c, F. Gutiérrez-Rodero a. Examen de salud en la población inmigrante: prevalencia de infección tuberculosa latente, hepatitis $\mathrm{B}$, hepatitis $\mathrm{C}$, infección VIH y sífilis. Enferm Infecc Microbiol Clin. 2003; 21: 540-2.

5. J.A. Casacante, J. Hueto. Tuberculosis como enfermedad ocupacional. An Sist Navar 2005 ; 28 : 1: $107-115$.

6. Gonzalez C, Araujo G, Agoglia R, Hernández S, Seguel I, Saenz C. Tuberculosis among health care workers. Medicina (B Aires). 2010;70(1):23-30

7. Sawanyawisuth K, Chaiear N, Sawanyawisuth K, Limpawattana P, Bourpoern J, Reechaipichitkul W, Takahashi K. Can workplaces be predictors for recent onset latent tuberculosis in health care workers?. Arch Intern Med. 2009 Jan 26;169(2):179-87.

8. Franchi A, Diana O, Franco G. Job-related risk of latent tuberculosis infection in a homogeneous population of hospital workers in a low incidence area. Am J Ind Med. 2009 Apr;52(4):297-303.

9. García-Guerrero1, A. Marco Mouriño2, P. Sáiz de la Hoya Zamácola3, E.J. Vera-Remartínez1 y Grupo de estudio PREVALHEP de prisiones* Estudio multicéntrico de prevalencia de infección tuberculosa latente en los internados en prisiones españolas Rev. esp. sanid. penit. 2010: 12: 79-85.

10. Swindells J.E, Aliyu S.H., Enoch D.A. and Abubakar I. Role of interferon-gamma relase assays in healthcare workers. Journ Hosp Infecc. 2009 73:2 (101-108).

11. Casas I, Latorre I, Esteve M, Ruiz-Manzano J, Rodríguez D, Prat C, et al. Evaluación de los Ensayos de liberación de interferón gamma en el diagnóstico de la infección tuberculosa reciente en el personal sanitario. PLoS One. 2009 Aug 24;4(8):e6686

12. Yoshiyama T, Harada N, Higuchi K, Nakajima Y, Ogata H. Estimation of incidence of tuberculosis infection in health-care workers using repeated interferon-gamma assays. Epidemiol Infect. 2009 Dec;137(12):1691-8.

13. Girardi E, Angeletti C, Puro V, Sorrentino R, Magnavita N, Vincenti D, Carrara S, Butera O, Ciufoli AM, Squarcione S, Ippolito G, Goletti D. Estimating diagnostic accuracy of tests for latent tuberculosis infection without a gold standard among healthcare workers. Euro Surveill. 2009 Oct 29;14(43). pii: 19373

14. Álvarez León EE, Espinosa Vega E, Santana Rodríguez OE, Pérez Arellano JL, Molina Cabrillana JM, Linertová R, et al. Efectividad y costes del Quantiferon-TB Gold para diagnosticar tuberculosis latente. Informes de Evaluación de Tecnologías sanitarias. SESCS. Num. 2007/02

15. Fox BD, Kramer MR, Mor Z, Preiss R, Rusanov V, Fuks L, et al. The QuantiFERON-TB-GOLD assay for tuberculosis screening in healthcare workers: a cost-comparison analysis. Lung. 2009 NovDec;187(6):413-9

16. Linertová R, Álvarez-León EE, García-Pérez L, Serrano-Aguilar P. Costs of QuantiFERON-TB Gold versus tuberculin skin test in Spanish healthcare workers. J Hosp Infect. 2010 May;75(1):52-5. Epub 2010 Mar 21.

17. Welbel SF, French AL, Bush P, De Guzman D, Weinstein RA. Protecting health care workers from tuberculosis: a 10-year experience. Am J Infect Control. 2009 Oct;37(8):668-73. Epub 2009 Apr 28.

18. Placidi D, Tonozzi B, Alessio L, Porru S. Tuberculin skin test (TST) survey among healthcare workers (HCWs) in hospital: a systematic review of the literatura. G Ital Med Lav Ergon. 2007 Jul-Sep;29(3 Suppl):409-11.

19. Nienhaus A. Tuberculosis in healthcare workers. Pneumologie. 2009 Jan;63(1):23-30. Epub 2009 Jan 9. Review.

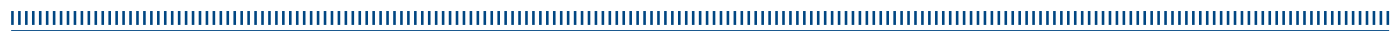

